\title{
Dislocation Jamming and Andrade Creep
}

\author{
M.-Carmen Miguel, ${ }^{1}$ Alessandro Vespignani, ${ }^{2}$ Michael Zaiser, ${ }^{3}$ and Stefano Zapperi ${ }^{4}$ \\ ${ }^{1}$ Departament de Física Fonamental, Facultat de Física, Universitat de Barcelona, \\ Avinguda Diagonal 647, E-08028 Barcelona, Spain \\ ${ }^{2}$ The Abdus Salam International Centre for Theoretical Physics, P.O. Box 586, 34100 Trieste, Italy \\ ${ }^{3}$ Center for Materials Science and Engineering, University of Edinburgh, King's Buildings, Sanderson Building, \\ Edinburgh EH93JL, United Kingdom \\ ${ }^{4}$ Istituto Nazionale per la Fisica della Materia, Unità di Roma 1 and Center for Statistical Mechanics and Complexity, \\ Dipartimento di Fisica, Università "La Sapienza", Piazzale A. Moro 2, 00185 Roma, Italy \\ (Received 8 May 2002; published 26 September 2002)
}

\begin{abstract}
We simulate the glide motion of an assembly of interacting dislocations under the action of an external shear stress and show that the associated plastic creep relaxation follows Andrade's law. Our results indicate that Andrade creep in plastically deforming crystals involves the correlated motion of dislocation structures near a dynamic transition separating a flowing from a jammed phase. Simulations in the presence of dislocation multiplication and noise confirm the robustness of this finding and highlight the importance of metastable structure formation for the relaxation process.
\end{abstract}

PACS numbers: 61.72.Lk, 68.35.Rh, 81.40.Lm

Andrade reported in 1910 that the creep deformation of soft metals at constant temperature and stress grows in time according to a power law with exponent $1 / 3$, i.e., $\gamma \sim t^{1 / 3}$, where $\gamma$ is the global strain of the material [1]. More generally, the creep curve usually follows the relation $\gamma=\gamma_{0}+\beta t^{1 / 3}+\kappa t$, where $\gamma_{0}$ is the instantaneous plastic strain, $\beta t^{1 / 3}$ is Andrade creep, and $\kappa t$ is referred to as linear creep [2]. This same behavior has since been observed in many materials with rather different structures leading to the conclusion that this should be a process determined by quite general principles, independent of most material specific properties. In crystalline materials, Andrade's and linear creep find their microscopic origin in the dynamics of dislocations, the topological defects responsible for their plastic deformation [2-4]. Plastic flow occurs only when the externally applied stress overcomes a threshold value, the yield stress of the material, such that large-scale dislocation motion may take place. Despite various arguments proposed within the dislocation literature [2,5-7], there is no general consensus on the basic mechanism to explain Andrade's law. Mott [5] attributed the power law to an athermal cooperative process taking place close to the yield stress, but his idea was not worked out. Later explanations have always focused on thermally activated processes [6,7] and rely on assumptions that may not be fully warranted [8].

Under the action of external stress, dislocations tend to glide cooperatively due to their mutual long-range elastic interactions, which can be attractive or repulsive. The anisotropic character of dislocation-dislocation interactions contributes at the same time to the formation of spatial dislocation patterns observed in transmission electron micrographs of plastically deformed metals [9]. As a result of these peculiar interactions, self-induced constraints build up in the system and the motion of disloca- tions may eventually cease. Small variations of the external load or the dislocation distribution can, however, enhance dislocation motion in a discontinuous manner. This gives rise to rather complex and heterogeneous spatiotemporal patterns of plastic flow, which have been observed experimentally in the form of slip lines and slip bands emerging on the surface of metals [10-12] or in the acoustic emission activity of ice crystals [13]. Other phenomena in plastically deforming crystals, such as hardening, fatigue, or plastic instabilities $[14,15]$, are further consequences of the dislocations intriguing behavior.

In this Letter, we study the temporal relaxation of a relatively simple dislocation dynamics model through numerical simulation. In particular, we consider the behavior of parallel straight edge dislocations moving in a single-slip system under the action of constant stress. We show that the model gives rise to Andrade-like creep at short and intermediate times for a wide range of applied stresses, without invoking thermally activated processes. At larger times the strain rate, which is proportional to the density of mobile dislocations, crosses over to a linear creep regime (steady rate of deformation), whenever the applied stress is larger than a critical threshold $\sigma_{c}$, or decays exponentially to zero. These results suggest that a possible interpretation of the creep laws could be found within the general scenario wrapping a dynamic phase transition from a flowing to a jammed dislocation phase. The "jamming" scenario has been recently proposed [16] to understand a broad class of nonequilibrium physical systems (granular media, colloids, supercooled liquids, foams) which, in spite of their differences, exhibit common properties such as slow dynamics and scaling features near the jamming threshold. When jammed, these systems are unable to explore phase space, but they can be unjammed by changing the stress, the density, or the 
temperature. To further explore the analogies of dislocation motion and these so-called jammed systems, we consider the influence of dislocation multiplication, due, for instance, to the activation of Frank-Read sources [2-4], and thermal-like fluctuations on the dynamics of the dislocations. Dislocation multiplication favors the rearrangements of the system and reduces $\sigma_{c}$, but it does not affect the initial power-law creep. The introduction of a finite effective temperature $T$ has a similar effect.

We consider a two-dimensional (2D) model representing a cross section of a single-slip oriented crystal where $N$ pointlike edge dislocations glide in the $x y$ plane along directions parallel to the $x$ axis. Dislocations with positive and negative Burgers vectors (the topological charge characterizing a dislocation) $\mathbf{b}_{n}=s_{n}(b, 0)$, where $s_{n}=$ \pm 1 are assumed to be present in equal numbers, and the initial number of dislocations is the same in every realization. Several 2D models containing similar basic ingredients have been proposed in the literature in the past few years [13,17-19]. An important feature common to these models is that dislocations interact with each other through the long-range elastic stress field they produce in the host material. An edge dislocation with Burgers vector $(b, 0)$ located at the origin gives rise to a shear stress $\sigma^{s}$ at a point $\mathbf{r}=(x, y)$ of the form [2-4]

$$
\sigma^{s}(\mathbf{r})=D b \frac{x\left(x^{2}-y^{2}\right)}{\left(x^{2}+y^{2}\right)^{2}},
$$

where $D=\mu / 2 \pi(1-\nu)$ is a coefficient involving the shear modulus $\mu$ and the Poisson ratio $\nu$ of the material. We further assume an overdamped dynamics in which the dislocation velocities are linearly proportional to the local forces. Accordingly, the velocity of the $n$th dislocation along the glide direction, if an external shear stress $\sigma$ is also applied, is given by

$$
\frac{\chi_{\mathrm{d}}^{-1} \boldsymbol{v}_{n}}{b}=s_{n} b\left[\sum_{m \neq n} s_{m} \sigma^{s}\left(\mathbf{r}_{n m}\right)+\sigma\right],
$$

where $\chi_{\mathrm{d}}$ is the effective mobility of the dislocations [20] and $\mathbf{r}_{n m} \equiv \mathbf{r}_{n}-\mathbf{r}_{m}$ is the relative position vector of dislocations $n$ and $m$. Periodic boundary conditions are imposed in the direction of motion (i.e., the $x$ axis). In order to take correctly into account the long-range nature of the elastic interactions [Eq. (1)], we sum the stress over an infinite number of images. This sum can be performed exactly, and the results are reported in Ref. [3]. When the distance between two dislocations is of the order of a few Burgers vectors, linear elasticity theory [i.e., Eq. (1)] breaks down. In these instances, phenomenological nonlinear reactions, such as the annihilation of a pair of dislocations, describe more accurately the real behavior of dislocations in a crystal [13,17-19]. In our model, we annihilate a pair of dislocations with opposite Burgers vectors when the distance between them is shorter than $y_{\mathrm{e}}$. In the following, we measure all lengths in units of $y_{\mathrm{e}}$, time in units of $t_{0}=y_{\mathrm{e}}^{2} /\left(\chi_{\mathrm{d}} D b^{3}\right)$, and stresses in units of $D b / y_{\mathrm{e}}$ (for $\mathrm{Cu}, y_{\mathrm{e}} \approx 1.6 \mathrm{~nm}[21], b=2.56 \AA$, and $\left.\mathrm{Db} / \mathrm{y}_{\mathrm{e}} \approx 1500 \mathrm{MPa}\right)$.

To analyze creep relaxation, we integrate numerically the $N$ coupled equations using an adaptive step size fifthorder Runge-Kutta algorithm. Simulations start from a configuration of $N_{0}$ dislocations randomly placed on a square cell of size $L$. We have considered two different box sizes $L=100 y_{\mathrm{e}}$ and $L=300 y_{\mathrm{e}}$, with initial numbers of dislocations $N_{0}=400$ and $N_{0}=1500$, respectively. We first relax the system until it reaches a metastable arrangement. During this relaxation, the dislocation density decreases due to annihilation processes until it is about $\rho_{0} \approx 0.01$ in nondimensional units. Next, we apply an external shear stress and let the system evolve [22]. The results are typically averaged over several (100-400) random initial configurations.

In Fig. 1, we report the plastic strain rate of the material, defined as $d \gamma / d t \sim \sum_{i} b_{i} v_{i}$, for different values of the applied stress. The strain rate decays as a power law, with an exponent close to $2 / 3$, in agreement with Andrade's law. For high stresses, the power-law relaxation is followed by a plateau indicating the onset of a linear creep regime. The crossover time increases as the stress decreases, and for stresses lower than $\sigma \simeq 0.0075-0.01$ the plateau disappears and the creep decays exponentially to zero. In Fig. 2, we display the steady-state strain rate as a function of stress. This plot suggests the presence of a nonequilibrium phase transition between a moving and a jammed stationary state controlled by the applied stress. Using $\sigma_{c}=0.0075$, we find $d \gamma / d t \sim\left(\sigma-\sigma_{c}\right)^{\beta}$ with $\beta=1.8 \pm 0.1$. Since the dislocation density in the steady-state regime is almost stress independent, it follows from Orowan's law that the stress exponent of the mean dislocation velocity $(\beta=1.8)$ differs from the

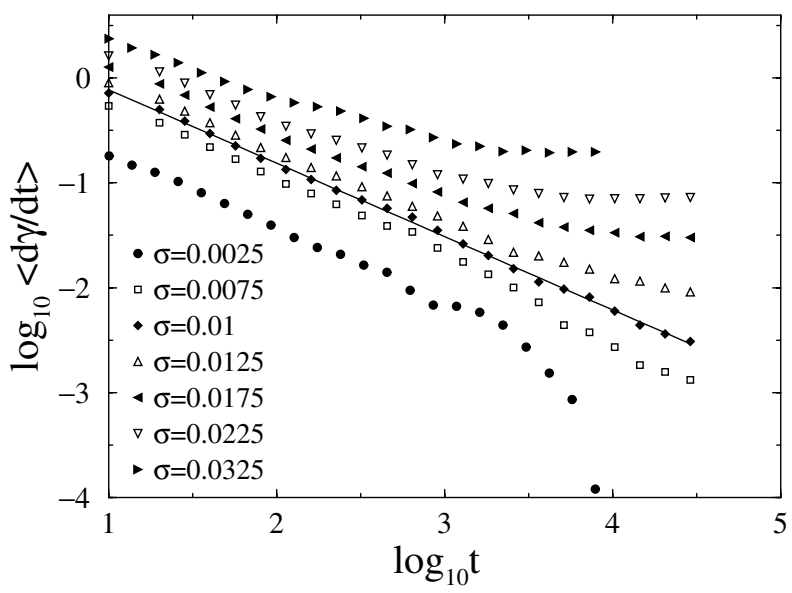

FIG. 1. The strain-rate relaxation for different applied stresses at $T=0$ for a system of size $L=100 y_{\mathrm{e}}$. The initial density of dislocations is around $1 \%$. The solid line is the best linear fit of the $\sigma=0.01$ curve and yields $d \gamma / d t \sim t^{-0.69}$. 


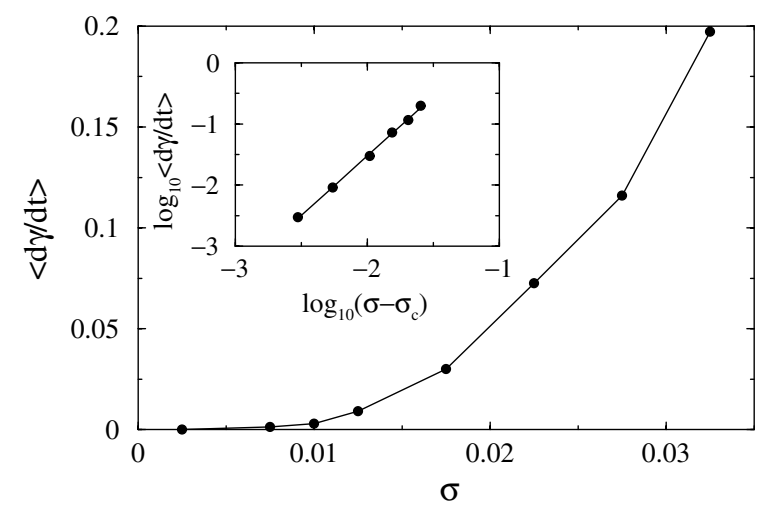

FIG. 2. The steady-state value of the strain rate as a function of the applied stress. The inset shows that the strain rate scales as $\left(\sigma-\sigma_{c}\right)^{\beta}$, with $\beta=1.8$.

stress exponent of the single-dislocation velocity (which is assumed to be 1). This illustrates the irreducibly collective character of dislocation dynamics which precludes any straightforward conclusions from singledislocation properties on the properties of the dislocation ensemble.

Within a jamming scenario, it appears natural to ask whether dislocation multiplication and thermal fluctuations affect the behavior of the system in a relevant way. During plastic deformation, in fact, new dislocations are created within the crystal, mainly through the activation of Frank-Read sources [2-4]. Since this mechanism cannot be directly simulated in a two-dimensional model, we employ a phenomenological procedure, introducing dislocation pairs with a rate $r$ proportional to the external stress. Similar multiplication mechanisms have been successfully used in the past $[13,17-19]$. Thermal fluctuations are accounted for by adding a Gaussian random force per unit length $\eta_{n}(t) / b$ to Eq. (2). The force has zero mean and its correlations are given by

$$
\left\langle\eta_{n}(t) \eta_{m}\left(t^{\prime}\right)\right\rangle=k_{B} T \chi_{\mathrm{d}}^{-1} \delta_{n, m} \delta\left(t-t^{\prime}\right),
$$

where $T$ is an effective temperature characterizing the strength of the fluctuations [23]. This random force could also mimic, as a first approximation, the influence of dislocation motion in other slip systems that may be active simultaneously in the material (see $[9,24]$ ).

We have performed a series of simulations for different multiplication rates and effective temperatures (measured in units of $\mathrm{Db} / k_{B}$ which is about $10000 \mathrm{~K}$ for $\mathrm{Cu}$ ) when the applied stress is close to the critical value (i.e., $\sigma=$ $0.0075)$. We used the same procedure as described previously and, given the additional fluctuations present in the strain rate, we focus our attention on the integrated plastic strain $\gamma$. As shown in Fig. 3, a linear creep regime appears after a crossover time which decreases with $r$ and $T$. Nevertheless, Andrade creep (i.e., $\gamma \sim t^{1 / 3}$ ) still persists at shorter times. A visual inspection of the dislocation arrangements during the deformation is useful to

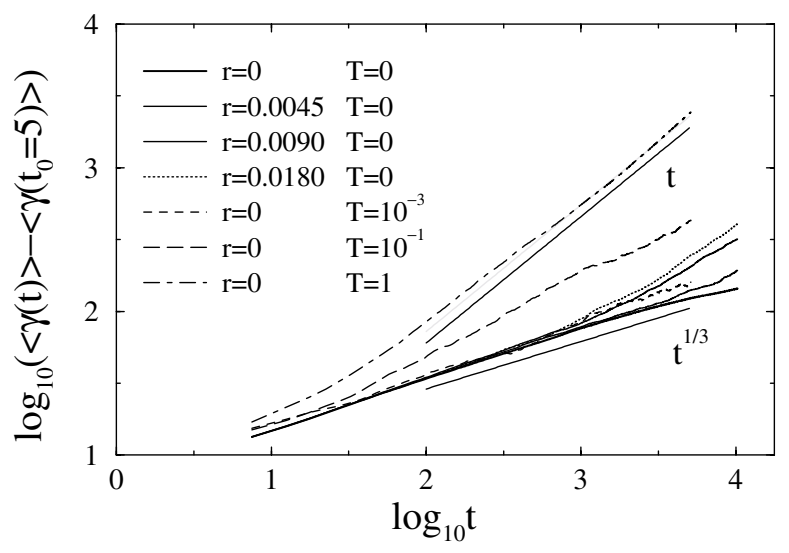

FIG. 3. The effect of the dislocation multiplication rate $r$ and of the effective temperature $T$ on the plastic strain in a system of size $L=100 y_{\mathrm{e}}$ with an applied stress $\sigma=0.0075$. Andrade creep $\left(\gamma \sim t^{1 / 3}\right)$ crosses over to linear creep $(\gamma \sim t)$ at large times, with a crossover depending on $r$ and $T$.

understand the origin of the various creep regimes. At low $T$, we observe the presence of slowly relaxing metastable structures as for $T=0$. On the other hand, at a much higher effective temperature (i.e., $T \simeq 1$ ) these structures have completely broken up, the flow of dislocations becomes fluidlike, and Andrade creep disappears. In order to substantiate quantitatively this statement, we measure the angular correlation between dislocations. For each dislocation pair with coordinates $\vec{r}_{i}$ and $\vec{r}_{j}$, we define $\theta$ as the azimuthal angle with respect to the $y$ axis of the vector $\vec{r}_{i j}$ [i.e., $\left.\theta \equiv \arccos \left(\hat{y} \cdot \vec{r}_{i j} /\left|\vec{r}_{i j}\right|\right)\right]$. Thus, two dislocations placed in the same wall have either $\theta \simeq 0$ or $\theta \simeq$ $\pi$, a dislocation dipole is characterized by $\theta \simeq \pi / 4$, $3 \pi / 4$, and two dislocations in a pileup yield $\theta \simeq \pi / 2$ [2-4]. The distribution $P(\theta)$ is obtained after averaging over all dislocation pairs in several realizations $(\sim 100)$ of the dynamics at a given instant. Figure 4 shows $P(\theta)$ for different values of $T$. At low effective temperatures, we observe five roughly equivalent peaks, corresponding to walls, dipoles, and pileup configurations. As the

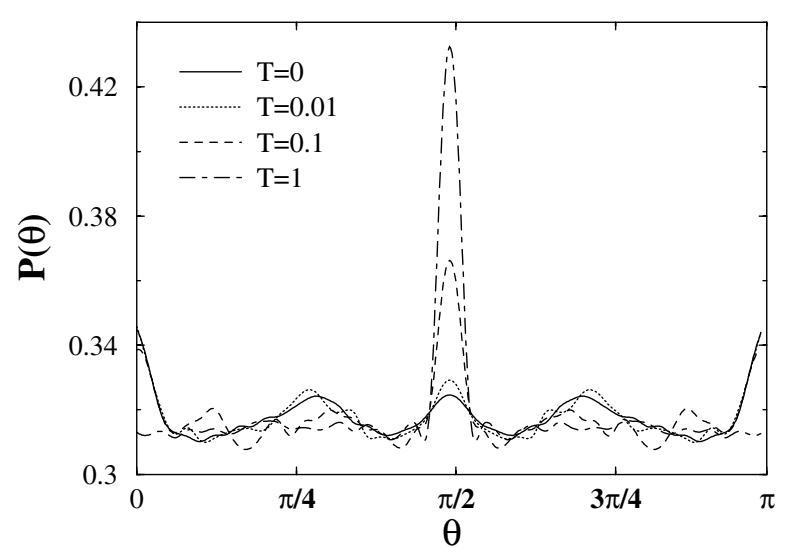

FIG. 4. The angle distribution as a function of the temperature at $\sigma=0.0075$. 
effective temperature increases, the peaks at $\theta=0, \pi$ and $\theta \simeq \pi / 4,3 \pi / 4$, corresponding to walls and dipoles, progressively disappear, while the $\theta=\pi / 2$ peak remains. This peak indicates that even in the hightemperature regime the dislocations are correlated in the $x$ direction, the only possible direction of motion. Apart from this, no other structure remains in the system.

In conclusion, Andrade's scaling and the creep curve appear to be controlled by a nonequilibrium phase transition. The effects of dislocation multiplication and effective temperature around the critical value $\sigma=0.0075$ smooth out this transition whose critical behavior is observed only as a transient crossing over to the supercritical phase, the linear creep regime, where dislocation structures are coherently moving with a velocity that grows with the applied stress. The transition originates from two fundamental properties of dislocation systems: (a) the ability of dislocations to form metastable jammed configurations and (b) long-range interactions which lead to collective dislocation motions and continuous rearrangements. For stress values below the critical threshold, dislocations cannot get around the self-induced constraints built up by their mutual (attractive/repulsive) interactions and are thus unable to further explore the configuration space, which is the signature of a jammed state [16]. Determining the critical stress value and characterizing its behavior (possible dependence on density and temperature) is of utmost importance for practical purposes, since it establishes the mechanical strength of crystalline materials. Evidently, plastic deformation of real crystals, where dislocations are flexible lines and in general more than one slip system is active, differs in many respects from our simple model. Nevertheless, both long-range interactions and jammed configurations are present in 3D dislocation systems - even if the latter may be made up of dislocation junctions rather than dipoles, multipoles, and walls. Therefore we believe that our basic scenario carries over to 3D situations.

We thank R. Pastor-Satorras for useful discussions. M.C.M. acknowledges financial support from the Ministerio de Ciencia y Tecnología (Spain).

[1] E. N. da C. Andrade, Proc. R. Soc. A 84, 1 (1910); 90, 329 (1914).

[2] J. Friedel, Dislocations (Pergamon, Oxford, 1967).

[3] J. P. Hirth and J. Lothe, Theory of Dislocations (Krieger, Malabar, Florida, 1992).

[4] F. R. N. Nabarro, Theory of Crystal Dislocations (Dover, New York, 1992).

[5] N. F. Mott, Philos. Mag. 44, 741 (1953).

[6] A. H. Cottrell, Philos. Mag. Lett. 73, 35 (1996); 74, 375 (1996); 75, 301 (1997).
[7] F. R. N. Nabarro, Philos. Mag. Lett. 75, 227 (1997).

[8] The power-law creep relaxation comes out by introducing a phenomenological hardening law and assuming that the strain produced by a thermal activation event is proportional to the activation volume - which may not be true for dislocations moving through a field of discrete obstacles as studied in J. G. Sevillano, E. Bouchaud, and L. P. Kubin, Scr. Metall. Mater. 25, 355 (1991); G. D'Anna, W. Benoit, and V. M. Vinokur, J. Appl. Phys. 82, 5983 (1997); S. Zapperi and M. Zaiser, Mater. Sci. Eng. A 309-310, 348 (2001).

[9] P. Hähner, K. Bay, and M. Zaiser, Phys. Rev. Lett. 81, 2470 (1998).

[10] R. Becker and E. Orowan, Z. Phys. 79, 566 (1932).

[11] V. Z. Bengus, S. N. Komnik, and O. B. Shititelman, Phys. Status Solidi 14, 215 (1966).

[12] H. Neuhäuser, in Dislocations in Solids, edited by F. R. N. Nabarro (North-Holland, Amsterdam, 1983), p. 319.

[13] M.-C. Miguel, A. Vespignani, S. Zapperi, J. Weiss, and J. R. Grasso, Nature (London) 410, 667 (2001).

[14] G. Ananthakrishna et al., Phys. Rev. E 60, 5455 (1999).

[15] G. D'Anna and F. Nori, Phys. Rev. Lett. 85, 4096 (2000).

[16] A. J. Liu and S. R. Nagel, Nature (London) 396, 21 (1998).

[17] J. Lepinoux and L. P. Kubin, Scr. Metall. 21, 833 (1987).

[18] R. J. Amodeo and N. M. Ghoniem, Phys. Rev. B 41, 6958 (1990); 41, 6968 (1990).

[19] I. Groma and G. S. Pawley, Philos. Mag. A, 67, 1459 (1993).

[20] The effective mobility of the dislocations may be controlled by thermal activation over atomic-scale obstacles (e.g., Peierls barriers, dislocation cores, atomic defects). In the low-stress high-temperature regime, this gives rise to a linear stress dependence of the dislocation velocity with a mobility $\chi_{\mathrm{d}} \propto \exp (-\Delta G / k T)$ that depends exponentially on temperature ( $\Delta G$ is the barrier height); see, e.g., U. F. Kocks, A.S. Argon, and M.S. Ashby, Prog. Mater. Sci. 19, 1 (1975). This temperature dependence may strongly affect the rate of creep relaxation, but does not change the nature of the $T=0$ nonequilibrium critical point underlying the power law.

[21] U. Essmann and H. Mughrabi, Philos. Mag. A 40, 731 (1979).

[22] Dislocation annihilation is significant during the initial relaxation at zero stress. Since this eliminates most close pairs, only little annihilation takes place during the subsequent creep simulation: The fraction of dislocations which annihilate after the stress is applied is typically about $2 \%-5 \%$ of the initial density only. Therefore our results, which have been obtained for a high dislocation density, can be scaled down to lower densities where dislocation annihilation is unimportant.

[23] We consider a model of rigid dislocations threading the whole sample. Thus, the thermal effects we have included may be relevant only in very thin samples, thicker specimens being captured by zero temperature results.

[24] I. Groma and B. Bako, Phys. Rev. Lett. 84, 1487 (2000). 